\title{
LE KYSTE DE LA VALLECULE : A PROPOS DE 2 OBSERVATIONS
}

\author{
I. HARIGA, A. MARDASSI, S. ZRIBI, A. CHARFEDDINE, M.B. AMOR, \\ O.B. GAMRA, CH. MBAREK, A. EL KHEDIM \\ SERVICE D'ORL ET DE CHIRURGIE CERVICO-FACIALE \\ HÔPITAL HABIB THAMEUR DE TUNIS
}

\begin{abstract}
Le kyste de la vallécule est une malformation laryngée rare pouvant parfois mettre en jeu le pronostic vital. Diagnostiqué et traité à temps, le risque de complications et de morbidité peut être écarté. Les auteurs rapportent 2 cas de kystes de la vallécule. La tomodensitométrie a confirmé la présence d'une formation kystique prenant origine au niveau de la vallécule. Une laryngoscopie directe a été faite pour les 2 patients sous anesthésie générale. Le premier malade a eu une résection endoscopique complète du kyste et le 2ème une large marsupialisation au Laser. L'examen anatomopathologique a confirmé la bénignité de la formation kystique dans les 2 cas.
\end{abstract}

Mots-clés : Kyste de la vallécule, laryngoscopie directe, résection endoscopique, marsupialisation.

\begin{abstract}
Vallecular cyst is a rare laryngeal anomaly that can have life threatening clinic presentation. However, when it is recognized and treated early, serious complications and morbidity can be avoided. The authors present 2 cases of vallecular cyst. A computed tomography (CT) scan confirmed the presence of a large cyst arising from the valleculae. A direct laryngoscopy was performed for the 2 patients under general anesthesia. Complete endoscopic excision was realized in one case and marsupialization of the cyst with laser was performed in the second case. Histologic examination supported the diagnosis of a benign cyst.
\end{abstract}

Keywords : Vallecular cyst, direct laryngoscopy, endoscopic excision, marsupialization.

\section{INTRODUCTION}

Les malformations kystiques du larynx sont relativement rares mais méritent d'être bien connues car elles peuvent poser des problèmes diagnostiques et thérapeutiques notamment dans un contexte d'urgence. La sévérité des symptômes est variable associant un stridor, une dyspnée obstructive, une dysphonie et des troubles de la déglutition. Le diagnostic repose sur l'examen clinique mais surtout sur l'endoscopie qui conditionnera la prise en charge ultérieure (1).

\section{OBSERVATIONS}

\section{Cas $n^{\circ} 1$ :}

II s'agit d'une patiente âgée de 14 ans sans antécédents pathologiques notables qui accuse depuis 2 mois une dysphagie mixte et une dysphonie sans autres signes associés. La laryngoscopie indirecte note une formation kystique transparente remontant dans l'oropharynx et l'obstruant en partie. La nasofibroscopie visualise une masse grossièrement arrondie prenant naissance au niveau de la vallécule gauche, et obstruant en partie l'oropharynx et l'hypopharynx. Une tomodensitométrie cervicale avait objectivé le caractère kystique de cette formation occupant l'étage sus-glottique, mesurant 2,4 x $3 \mathrm{~cm}$ de grand axe et obstruant la quasi-totalité de la lumière de l'oropharynx (fig. 1). Au cours du geste opératoire, une trachéotomie première de sécurité a été réalisée. La laryngoscopie directe montrait que la masse prenait naissance au niveau de la vallécule gauche et qu'elle empiétait sur la face linguale et le bord libre gauche de l'épiglotte. Le décollement du kyste au niveau de sa base d'implantation permettait son exérèse en totalité (fig.2). Les suites opératoires étaient simples et la décanulation

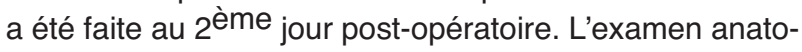
mopathologique définitif a conclu à un kyste valléculaire bénin revêtu d'une muqueuse de type respiratoire. Le recul actuel est de six mois.

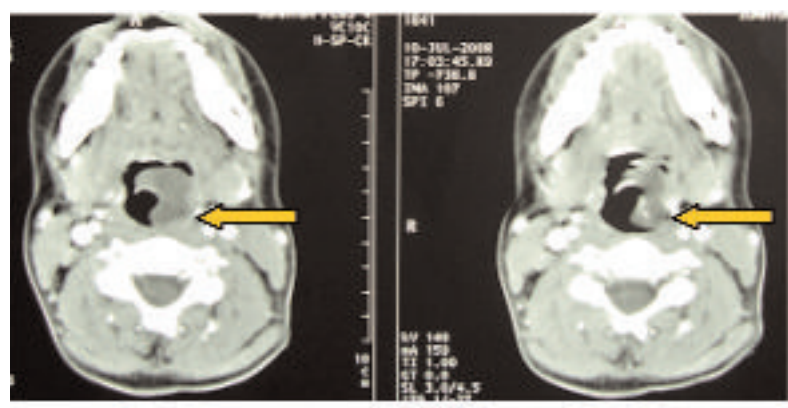

Fig. 1: TDM cervicale en coupes axiales montrant la masse kystique à point de départ valléculaire gauche et comblant en partie l'oropharynx. 

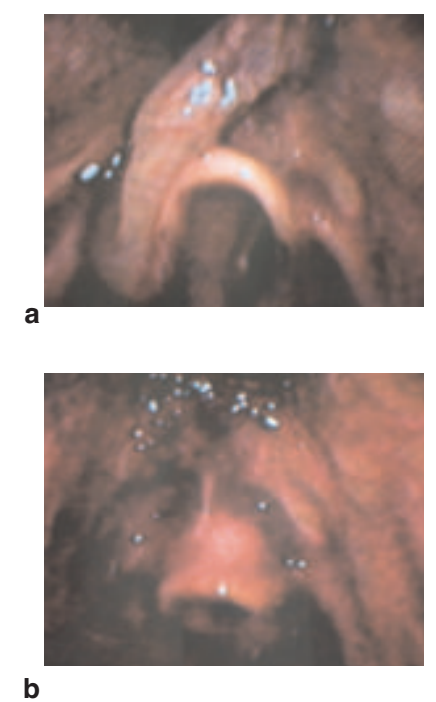

Fig. 2 : Décollement endoscopique du kyste au niveau de sa base d'implantation (a) et vue endoscopique post-opératoire (b).

\section{Cas $\mathrm{n}^{\circ} 2$ :}

II s'agit d'un patient âgé de 35 ans sans antécédents pathologiques qui a consulté pour une dysphagie haute d'aggravation progressive évoluant depuis 5 mois associée à une voix étouffée sans dyspnée. Une nasofibroscopie a noté la présence d'une grosse formation kystique prenant origine au niveau de la vallécule droite et obstruant la quasi-totalité de l'oropharynx(fig. 3). La tomodensitométrie cervicale avait objectivé une image arrondie bien limitée mesurant $2,7 \times 3 \mathrm{~cm}$ de grand axe de densité liquidienne se réhaussant en couronne périphérique fine après injection de produit de contraste développée au niveau de la vallécule droite (fig. 4). La laryngoscopie directe faite après une trachéotomie première a visualisé la grosse formation kystique qui était bien limitée recouverte d'une muqueuse saine et qui obstruait presque totalement l'espace rétro-basilingual. L'incision du kyste a permis d'évacuer un liquide mucoïde épais. Une résection du dôme saillant du kyste a été ensuite faite au Laser. Les suites opératoires étaient simples et la décanulation a été faite au 3ème jour post-opératoire. L'examen anatomopathologique définitif a conclu à un kyste remanié de la vallécule sans signes histologiques de malignité. Le recul actuel est de 3 ans et demi.

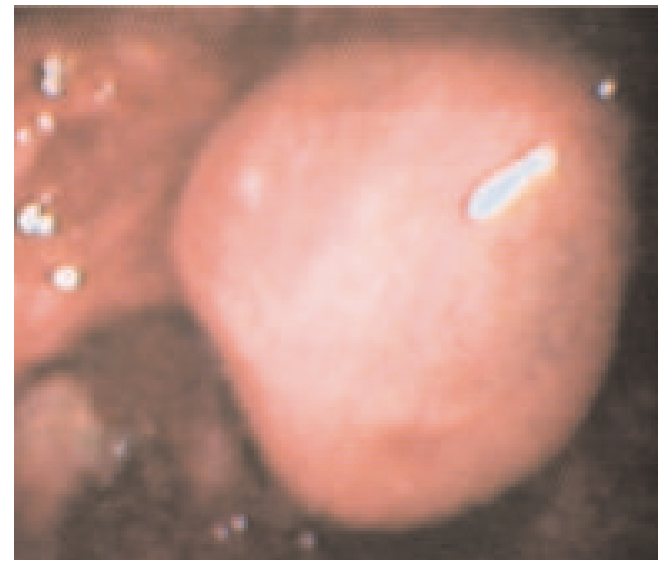

Fig. 3 : Vue endoscopique de la masse kystique de la vallécule

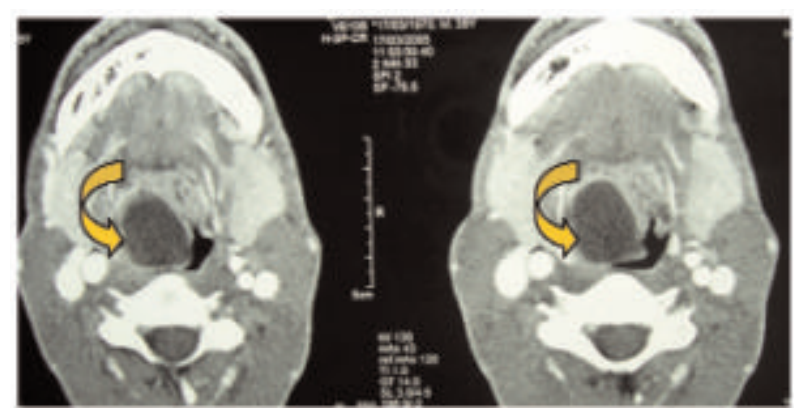

a

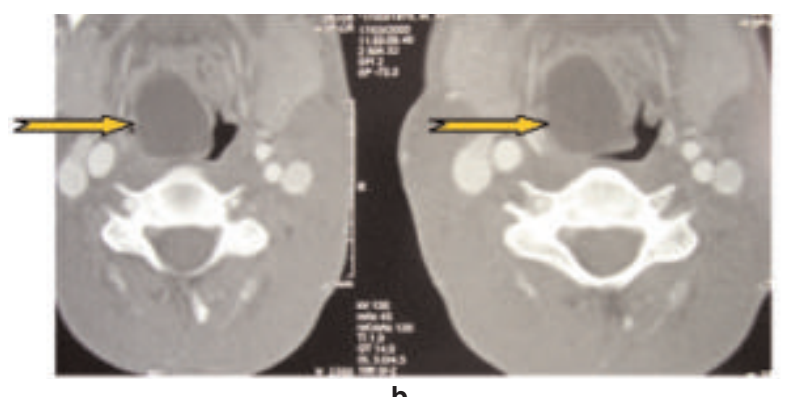

b

Fig. 4: TDM cervicale en coupes axiales, en fenêtre parenchymateuse (a) et en fenêtre osseuse (b) montrant une volumineuse formation kystique arrondie bien limitée de densité liquidienne se développant à partir de la vallécule droite et comblant la quasi-totalité de l'espace oropharyngé.

\section{DISCUSSION}

Les kystes du larynx sont classés en kystes sus-glottiques, glottiques et sous-glottiques selon leur symptomatologie et de leurs étiologies différentes $(2,3)$. On distingue deux mécanismes étiopathogéniques quant à leur formation (4). Les kystes de type canalaire ou rétentionnel sont les plus répandus et sont ubiquitaires (2). Ils proviennent de l'obstruction des canaux des glandes muqueuses par une inflammation ou suite à un traumatis- 
me $(5,6)$. Leur paroi est recouverte d'un épithélium identique à celui du canal excréteur des glandes (3). En revanche, les kystes sacculaires se développent à l'étage sus-glottique, dans l'aire du saccule laryngé. Ils sont plus volumineux que les précédents, leur paroi est bordée d'un épithélium de type respiratoire et peuvent être congénitaux ou acquis $(2,7,8,9)$.

Les kystes épiglottiques et valléculaires sont de type rétentionnel (10). L'inflammation chronique, aboutissant à I'obstruction des glandes à mucus en serait la cause principale $(4,11)$. Toutefois, une étiopathogénie congénitale a également été envisagée car certains kystes épiglottiques ont été décrits chez des nouveau-nés de 6 semaines (8). Ils sont parfois multiples et leur taille varie de $2 \mathrm{~mm}$ à 3 $\mathrm{cm}$ (1). Habituellement asymptomatiques et découverts lors d'un examen systématique, ils peuvent parfois être responsables de paresthésies pharyngées à type de sensation de corps étranger $(4,12)$. Rarement ils peuvent devenir obstructifs et être responsables de dyspnée, voire d'asphyxie aiguë $(1,4,11,13,14)$. Ce phénomène se produit surtout lors d'épisodes de surinfection. Chez l'enfant, les kystes peuvent se révéler par un stridor, ou par une détresse respiratoire, en raison de l'étroitesse de la filière laryngée (12). En raison de sa localisation anatomique, le kyste de la vallécule est une cause rare, mais classique d'obstruction des voies aériennes supérieures et de décès du nouveau-né $(13,14,15)$. La dysphagie et la douleur accompagnent rarement ces kystes $(8,11)$. Un cas de syndrôme d'apnée du sommeil lié à un kyste de la vallécule a été rapporté par des auteurs japonais (16).

L'examen fibroscopique est pratiqué de première intention. II est préférable à la laryngoscopie directe qui peut méconnaître un kyste basilingual ou valléculaire chargé par la lame du laryngoscope $(1,2)$. Le diagnostic est, ensuite, posé par l'endoscopie laryngée sous anesthésie générale qui retrouve, dans une vallécule ou sur l'épiglotte, le plus souvent au niveau de la face linguale, une tuméfaction claire, régulière, dont la surface est parcourue par un fin lacis vasculaire $(1,4,8,14)$.

L'échographie laryngée et le scanner peuvent apporter des précisions, surtout s'il existe un doute avec une laryngocèle ou une pathologie néoplasique (15). L'échographie confirme le caractère liquidien de la lésion $(1,8,13)$. Le scanner demeure toutefois l'examen essentiel en objectivant une masse sus-glottique régulière, homogène, hypodense, ne prenant pas le contraste (2). L'imagerie par résonance magnétique peut également être pratiquée et montre une lésion de même intensité que le liquide céphalorachidien en T1 et T2 $(4,15)$. Cependant, elle ne semble pas apporter d'éléments supérieurs à ceux du scanner, qui devra rester l'examen de première intention $(4,8)$.

Le traitement des kystes laryngés est identique à celui des laryngocèles $(3,8)$. En présence d'une dyspnée aiguë, le traitement relève d'une trachéotomie en urgence
$(4,7,11)$. En dehors de ce cas, la chirurgie se fait de façon réglée. Deux options se présentent alors : le traitement par voie endoscopique et l'abord externe $(4,8)$. Lors d'un traitement endoscopique, plusieurs attitudes peuvent être adoptées (2) : une incision simple du kyste avec aspiration de son contenu, cette technique expose à une plus grande fréquence de récidives ; une marsupialisation instrumentale ou au laser $\mathrm{CO} 2(2,5,7,8)$; et enfin, l'exérèse complète du kyste (14). Celui-ci est alors disséqué jusqu'à son orifice qui est lié et les reliquats peuvent être vaporisés au laser $(4,6)$. Le risque de récurrence est réel et plus fréquent en cas de marsupialisation. Plusieurs séries l'ont rapporté, toutefois, aucun chiffre précis n'a été publié $(2,5,7,8)$. Le traitement des kystes de la vallécule est pratiqué sous laryngoscopie en suspension en prenant bien soin de ne pas charger le kyste avec la spatule du laryngoscope de façon à exposer le maximum de surface. En cas de dyspnée importante ou de difficultés d'exposition de la glotte, il peut être utile au préalable d'affaisser le kyste par ponction directe à l'aide d'une aiguille de gros calibre (1). L'intervention consiste alors à effectuer l'exérèse de la poche kystique le plus complètement possible. Si cela s'avère impossible, une large marsupialisation de la poche kystique peut être tentée mais le risque de récidive est important $(2,6,7,11)$. La voie externe fait appel à une incision cervicale horizontale latérale. Le bord supérieur du cartilage thyroïde est repéré et la membrane thyrohyoïdienne incisée à ce niveau. Le kyste est alors disséqué et sa base est liée $(4,8)$. Un abord chirurgical externe est recommandé pour les kystes volumineux (2). Chez l'enfant, on pratique en première intention une incision simple du kyste ou une marsupialisation qui peut être répétée en cas d'échec. En l'absence de résultat satisfaisant, un abord chirurgical externe est conseillé (8). Cette voie est volontiers précédée d'une trachéotomie première afin de prévenir l'inondation de l'arbre trachéobronchique lors de la rupture peropératoire du kyste(10). Elle prévient également les troubles respiratoires liés à un éventuel œdème laryngé postopératoire (7).

\section{CONCLUSION}

Les kystes de la vallécule sont de type rétentionnel et sont rares. Leur manifestation est le plus souvent bénigne, mais il peut exister des formes graves, rapidement asphyxiques, et ce, de façon totalement imprévisible ce qui justifie le traitement de tout kyste diagnostiqué. Le diagnostic est porté par l'endoscopie laryngée. La résection endoscopique pose le problème de récidive de ces kystes d'où une surveillance régulière s'avère indispensable. 


\section{REFERENCES}

1) Crozat-Teissier $N$, Van Den Abbeele T. Malformations congénitales du larynx. EMC-Oto-rhino-laryngologie 2005; 2 : 218-29.

2) Evans D. Saccular cyst of the larynx. Otolaryngol Head Neck Surg 2003; 128:303-4

3) Raveh E, Inbar E, Shvero J, Feinmesser R. Huge saccular cyst of the larynx: a case report. J Laryngol Otol. 1995; 109: 653-6.

4) Righini C, Kadaoui H, Morel N, Lerena C, Reyt E. Stridor in a newborn caused by a congenital laryngeal saccular cyst. Intern J Pediatr Otorhinolaryngol Extra 2006; 1: 145-9.

5) Craig B. Laryngeal Saccular Cyst. Diagnostic and Therapeutic Endoscopy 2000; 6: 91-4.

6) Gallivan G, Gallivan H, Eitnier C. Dual Intracordal Unilateral Vocal Fold Cysts: A Perplexing Diagnostic and Therapeutic Challenge. J Voice 2008; 22: 119-24.

7) Lim J, Hellier W, Harcourt J, Leighton S, Albert D. Subglottic cysts: the Great Ormond Street experience. Intern J Pediatr Otorhinolaryngol 2003; 67: 461-5. 8) Thomassin J M, Epron J P, Robert D. Kystes du larynx et laryngocèles. Traité d'Oto-rhino-laryngologie 1996 : 20-695-A-10.

9) Busuttil $M$, Desbrière $R$, Chau $C$ et al. Kyste congénital de la vallécule : intérêt d'une approche multidisciplinaire. J Gyn Obst Biol Repr 2004; $33: 84$

10) Kimura M, Nakashima M, Nito T, Tayama N. Tonsillar cyst of the false vocal cord. Auris Nasus Larynx 2007; 34: 111-3.

11) Velepica M, Sainac G, Lahb K, Velepica MM, Starcevica R, Pedisica D. Congenital laryngeal cyst: one or two cysts. Intern J Pediatr Otorhinolaryngol 2003; $67: 283-5$

12) Beutner D, Preuss SF, Quante G, Klussmann JP, Damm M, Hüttenbrink KB. Vallecular cyst as a rare cause of progressive inspiratory stridor. Laryngorhinootologie 2006; 85 : 441-3.

13) Markus $C$, Bernhard $P$, Thomas $L$. Acute apnea caused by an epiglottic cyst. Intern J Pediatr Otorhinolaryngol 1998; 42: 271-6.

14) Tosun $F$, Söken $H$, Ozkaptan $Y$. Saccular cyst in an infant: an unusual cause of life-threatening stridor and its surgical treatment. Turk J Pediatr. 2006; 48 :17880

15) Cuillier F , Testud R, Samperiz S, Fossati P. Découverte anténatale à 25 semaines d'aménorrhée et prise en charge néonatale d'un kyste de la vallécule. Ann Otolaryng Chir Cerv Fac 2002; 119 : 293-5.

16) Lee L, Fang T, Li H Y. A Supraglottic Laryngeal Cyst Causing Obstructive Sleep Apnea Syndrome. Otolaryng Head Neck Surg 2005; 131 : 311. 